\title{
Larger Two-Dimensional Photonic Band Gaps
}

\author{
Cheryl M. Anderson and Konstantinos P. Giapis* \\ Division of Chemistry and Chemical Engineering, California Institute of Technology, Pasadena, California 91125
}

(Received 23 May 1996)

\begin{abstract}
Absolute photonic band gaps in two-dimensional square and honeycomb lattices of circular crosssection rods can be increased by reducing the structure symmetry. The addition of a smaller diameter rod into the center of each lattice unit cell lifts band degeneracies to create significantly larger band gaps. Symmetry breaking is most effective at filling fractions near those which produce absolute band gaps for the original lattice. Rod diameter ratios in the range 0.1-0.2 yield the greatest improvement in absolute gap size. Crystal symmetry reduction opens up new ways for engineering photonic gaps. [S0031-9007(96)01328-2]
\end{abstract}

PACS numbers: 42.70.Qs, 41.90.+e

The last few years have witnessed an ongoing search for periodic dielectric structures which give rise to a photonic band gap $(\mathrm{PBG})-\mathrm{a}$ region of the frequency spectrum where propagating modes are forbidden. These "photonic crystals" could alter radiation-matter interactions and thus improve the efficiency of optical devices by controlling spontaneous emission [1]. Applications of these crystals in semiconductor lasers and solar cells [1], and high-quality resonant cavities and filters [2] have been proposed. Although three-dimensional (3D) PBG crystals suggest the most interesting ideas for novel applications, two-dimensional (2D) structures could also find several important uses, as a result of their strong angular reflectivity properties over a wide frequency band. For example, 2D PBG crystals with absolute band gaps provide a large stop band for use as a feedback mirror in laser diodes [3]. Photonic gaps at visible to near-infrared (IR) wavelengths could have the widest impact in applications. As the band gap frequency is directly related to the size of the scattering elements comprising the lattice, a near-IR band gap requires features with dimensions in the submicron size regime. Fabricating 3D periodic structures in this regime poses an overwhelming challenge, despite progress in microfabrication technology. Perhaps for this reason attention has been drawn towards 2D lattice structures. The successful fabrication of 2D crystals with near-IR band gaps has been recently reported $[4,5]$.

The larger a $\mathrm{PBG}$ is, the greater the forbidden region of the frequency spectrum. Thus, it is essential to design crystal structures with the largest PBGs possible for a given dielectric contrast. For two different crystals possessing absolute band gaps of equal size, it may be advantageous from a fabrication standpoint to choose the one that has the band gap occurring at the higher nondimensionalized frequency, $\omega a / 2 \pi c$, where $\omega$ is the frequency, $a$ is the lattice constant, and $c$ is the speed of light in vacuum. For a given filling fraction, the feature size scales with $a$; thus, the crystal with the higher $\omega a / 2 \pi c$ should be easier to fabricate. But how does one sift through the countless geometrical arrangements to select manufacturable structures with large band gaps in the desired frequency region?
Theoretical calculations are necessary, albeit a formidable task in view of the numerous structures to model with many variational parameters (e.g., lattice type, filling fraction, shape of filling element). A rational approach towards the design of photonic crystals would be indispensable bruteforce computation.

Overlapping bands in a photonic band diagram constitute a degeneracy that can be lifted by symmetry breaking. Consider, for example, the 3D face-centered-cubic crystal, which does not possess a full PBG. By decreasing its symmetry through the introduction of a two-point basis set (which produces the diamond structure), a degeneracy in the bands is lifted and a full PBG is obtained [6]. Previous studies of symmetry breaking by varying the shape [7] or size [8] of the cylinders comprising the lattice failed to produce 2D structures having a larger PBG than the crystal with symmetric rods. However, we will show in this Letter that symmetry breaking through the addition of a different size rod into the lattice unit cell can be effective in producing larger PBGs in 2D crystals.

The first structure examined consists of two interpenetrating square lattices of the same lattice constant $a$, displaced with respect to each other by $\mathbf{a}=\frac{1}{2} a\left(\hat{\mathbf{x}}_{1}+\hat{\mathbf{x}}_{2}\right)$, where $\hat{\mathbf{x}}_{1}$ and $\hat{\mathbf{x}}_{2}$ are the primitive lattice vectors. The crystal is composed of infinite length circular rods of diameter $d_{1}$ at the corners of a square lattice, and diameter $d_{2}$ at the center of each unit cell. This arrangement forms a new structure when $d_{1} \neq d_{2}$, which we name the "reduced-symmetry square lattice." Both the single-rod and the reduced-symmetry square lattices belong to the plane group $\mathrm{p} 4 \mathrm{~mm}$, with all the unit cell elements having $4 \mathrm{~mm}$ symmetry [9]. Symmetry reduction is accomplished by increasing the number of rods in the primitive unit cell. All rods are assumed to be made of the same dielectric material, and are embedded in a different dielectric background. The ratio of the two rod diameters, $\beta=d_{2} / d_{1}$, critically affects the gap size.

The electromagnetic frequency spectra of the dielectric crystals have been calculated using the plane-wave expansion technique, described in detail in the literature $[6,10$, 11]. The results were obtained using 729 plane waves for 
the square lattice, and 1225 plane waves for the reducedsymmetry one. When using 1757 plane waves, the band frequencies differed from those calculated with fewer plane waves by a maximum of $0.8 \%$. Most bands differed by less than $0.5 \%$. Thus, we believe that all results herein are accurate to within at least $1 \%$ of their true values.

We start with the single-rod square lattice of air holes in a dielectric material, whose photonic properties have been previously studied by several groups [12-14], each reporting that band gaps for each of the two orthogonal polarizations occur. However, there has been some discrepancy as to whether an absolute PBG is present. Our calculations for the square lattice when the background material has a dielectric constant $\epsilon_{b}=11.4\left(\epsilon_{\mathrm{GaAs}}\right.$ at $\left.\lambda \approx 1.5 \mu \mathrm{m}\right)$ are summarized in Fig. 1, where nondimensionalized frequencies are plotted as a function of the filling fraction, $f$. The "gap map" shows two gaps for each of the $H$ and $E$ polarizations, with the upper gaps overlapping from filling fractions of 0.68 to 0.79 to produce an absolute band gap. The absolute gap is bounded on the lower side by the $H$-polarization gap boundary, and on its upper side by the $E$-polarization gap. A maximum gap of $\Delta \omega=0.0188(2 \pi c / a)$ occurs when $f=0.77$.

Our aim is to rationally modify this structure to enlarge the absolute band gap. The square lattice gap map clearly indicates that the $H$-polarization gap drops off sharply for $f \geq 0.77$, thus limiting the overlap with the $E$-polarization gap. Band structure analysis shows that the second and third bands for $H$ polarization (those that bound the higher frequency gap in Fig. 1) are degenerate at the $\mathbf{M}$ point of the Brillouin zone at large filling fractions, closing the band gap. If this band degeneracy can be lifted while maintaining (or increasing) the size of the $E$-polarization gap, a larger absolute band gap will ensue. We will show that this occurs by reducing the symmetry of the square lattice.

When a smaller diameter rod is placed at the center of each square unit cell, the crystal symmetry is reduced. Af-

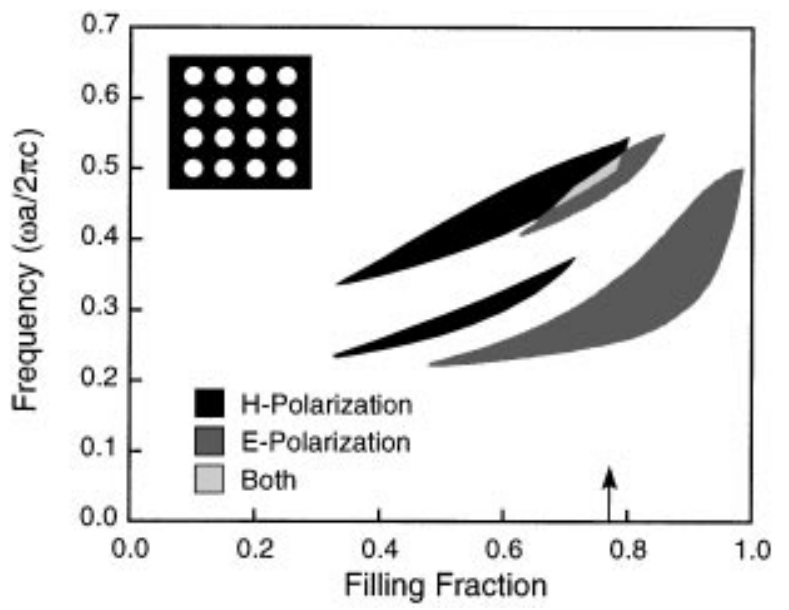

FIG. 1. Gap map for the single-rod square lattice of air holes in a background dielectric $\left(\epsilon_{b}=11.4\right)$. An absolute band gap occurs where the upper two polarization gaps overlap. The maximum gap occurs at $f=0.77$ (indicated by arrow). ter extensive calculations, we have found that values of $\beta$ between 0.1 and 0.2 provide the most dramatic improvement in the size of the absolute band gaps. Figure 2 shows the gap map for the reduced-symmetry square lattice when $\beta=0.16$. Introducing the smaller diameter rod does increase the size of the upper $H$-polarization gap. Remarkably, it also greatly enlarges the upper $E$-polarization gap. Thus, the overlap between the two gaps increases, resulting in a much larger absolute band gap. The maximum photonic gap occurs for $\beta=0.16$ and $f=0.793$. With a maximum gap width of $\Delta \omega=0.0548(2 \pi c / a)$, this band gap is nearly 3 times larger than the best value obtained for the single-rod square lattice case. It is noteworthy that the latter structure possesses no absolute gap at $f=0.793$. Symmetry breaking has introduced a large absolute photonic gap where none existed before.

Symmetry reduction may not be effective for all filling fractions. Figure 3 shows the effect of the parameter $\beta$ on the size and position of the band gaps for $f=0.675$. The band gaps for the single-rod square lattice are obtained when $\beta=0$ or, equivalently, unity [15]. It is seen that as the lattice moves away from the equal diameter case, the upper $H$-polarization gap tends to increase in size, reaching a maximum at an intermediate value of $\beta$. There is a region in the middle of the plot where the lower $\mathrm{H}$ polarization gap disappears at roughly the same $\beta$ where a new higher gap appears. This is a result of the equivalence of the two lattices when $\beta$ is either 0 or 1 . Two $E$ polarization gaps exist, but they do not overlap with the $H$-polarization gaps. Thus, symmetry breaking in this case does not help to produce an absolute band gap.

However, at a larger value $f=0.793$, a much more complex gap map unfolds as a result of symmetry breaking, as shown in Fig. 4. Several gaps in both $E$ and $H$ polarizations appear and disappear as $\beta$ is varied. However, in contrast to Fig. 3 , the second $E$ - and $H$-polarization

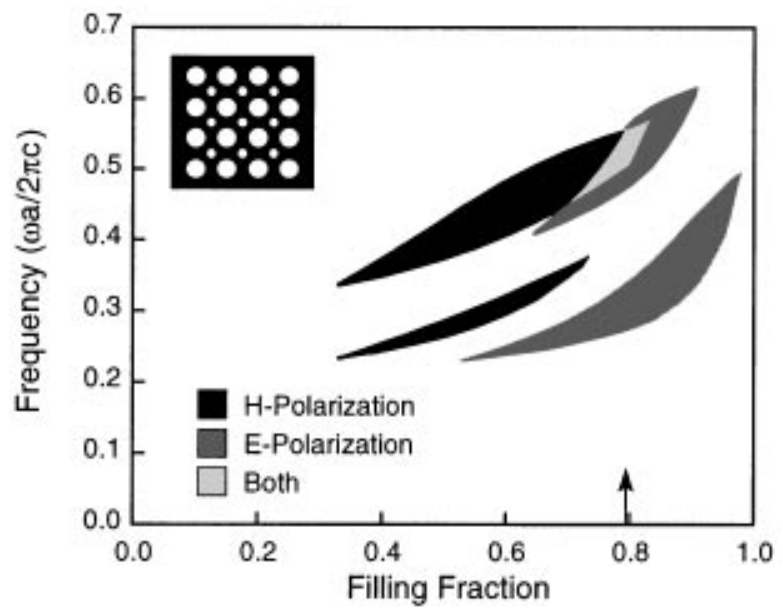

FIG. 2. Gap map for the reduced-symmetry square lattice $(\beta=0.16)$ of air holes in a background dielectric $\left(\epsilon_{b}=11.4\right)$, showing a significantly larger absolute band gap than that for the single-rod lattice. The maximum gap occurs at a filling fraction of 0.793 (arrow). 


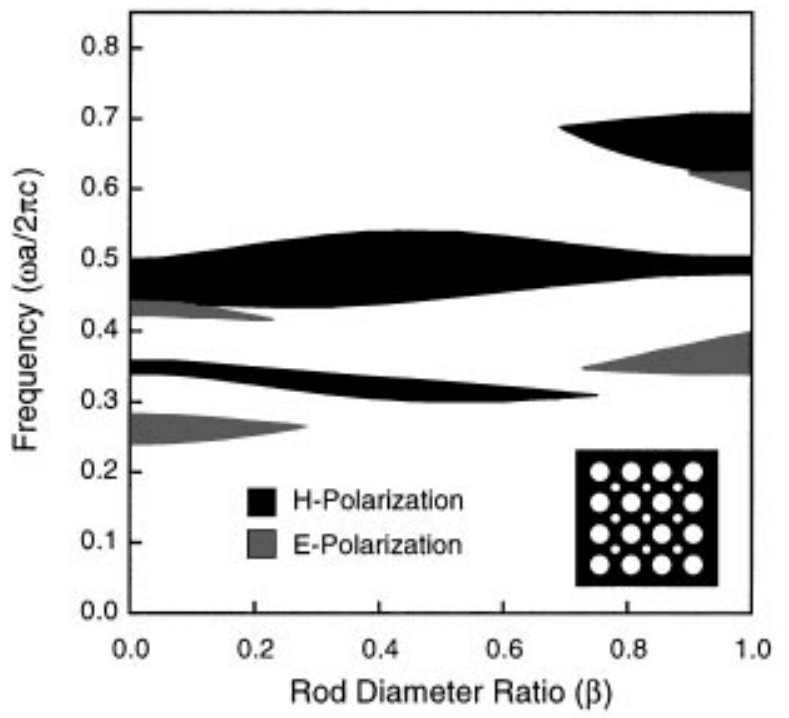

FIG. 3. Gap map for the reduced-symmetry square lattice $(f=0.675)$ of air holes in a background dielectric $\left(\epsilon_{b}=\right.$ 11.4), showing the effect of changing the rod diameter ratio on gaps for the two orthogonal light polarizations. No overlap exists between the $H$ - and $E$-polarization gaps.

gaps both increase and overlap more as $\beta$ becomes larger. Thus, a new absolute gap forms which reaches a maximum at $\beta=0.16$. In addition, another absolute gap forms at $\beta \approx 0.5$, albeit of smaller magnitude.

Both the filling fraction and the size of the symmetry breaking element are important in dictating which structures will possess absolute PBGs. Many band gaps close or open at filling fractions where the rods begin to overlap [16]. Adding a small rod in each unit cell may create

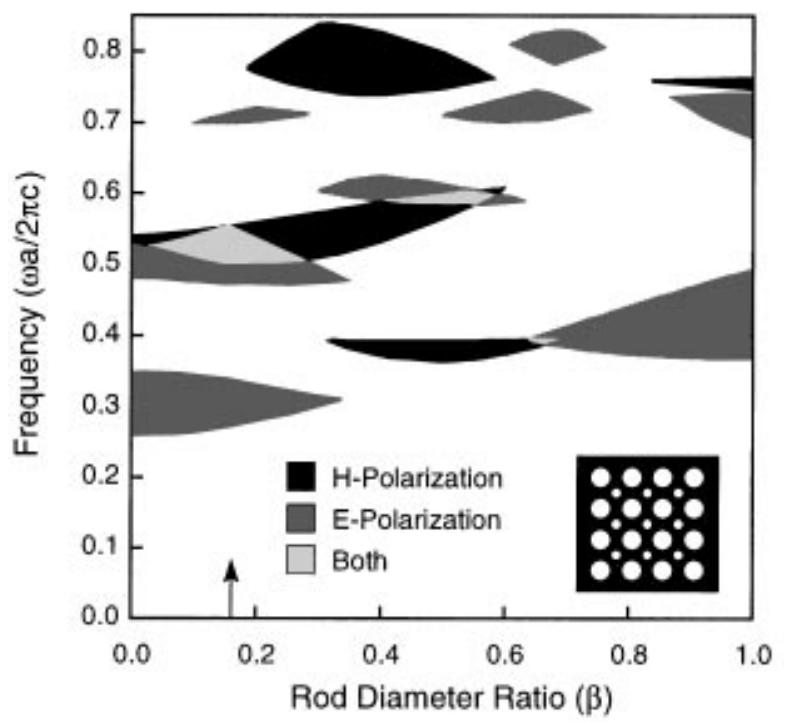

FIG. 4. Gap map for the reduced-symmetry square lattice $(f=0.793)$ of air holes in a background dielectric $\left(\epsilon_{b}=11.4\right)$ as a function of $\beta$. Symmetry reduction at the larger filling fraction opens up many new gaps for both polarizations, some of which do overlap to produce absolute band gaps. The largest absolute gap occurs at $\beta=0.16$ (arrow). larger absolute band gaps because the filling fraction is increased without disrupting the lattice connectivity.

The honeycomb lattice of dielectric rods in air is a promising candidate for the fabrication of a PBG crystal in the visible to near-IR because it exhibits a very large absolute gap (at a high non-dimensionalized frequency) that extends over a wide range of filling fractions [17]. Symmetry breaking can lead to even larger absolute band gaps when another rod is placed in the center of the honeycomb cell. The honeycomb structure itself can be viewed as an extreme case of asymmetry in the triangular lattice. Both the triangular and honeycomb lattices have plane group symmetry $p 6 \mathrm{~mm}$; however, the rod symmetry $(6 \mathrm{~mm})$ of the former lattice is reduced to $3 \mathrm{~m}$ in the latter [9]. The symmetry of the triangular lattice can be reduced by changing the diameter of the rod inside one hexagonal unit cell (Fig. 5 inset), yielding a new lattice. This "reducedsymmetry triangular lattice" is comprised of both $3 m$ and $6 m m$ symmetry rods. Here, the ratio of the rod diameters is defined as $\beta=d_{2} / d_{1}$, where the index 1 refers to the rods on the cell edge, and index 2 refers to the center rod. The honeycomb lattice is generated when $\beta=0$, and the triangular lattice when $\beta=1$. All calculations for the reduced-symmetry triangular lattice were performed using 1261 plane waves, to better than $1 \%$ accuracy.

For the reduced-symmetry triangular lattice of dielectric rods in air, adjusting the value of $\beta$ has the opposite effect on the gaps of the two orthogonal polarizations. The $\mathrm{H}$ polarization gaps tend to decrease monotonically as $\beta$ increases, while the $E$-polarization gaps reach a maximum for an intermediate value of $\beta$. This trend is expected, since the high dielectric constant material is now located in the rods. A rod diameter ratio is sought which takes advantage of the increasing size of the $E$-polarization gap,

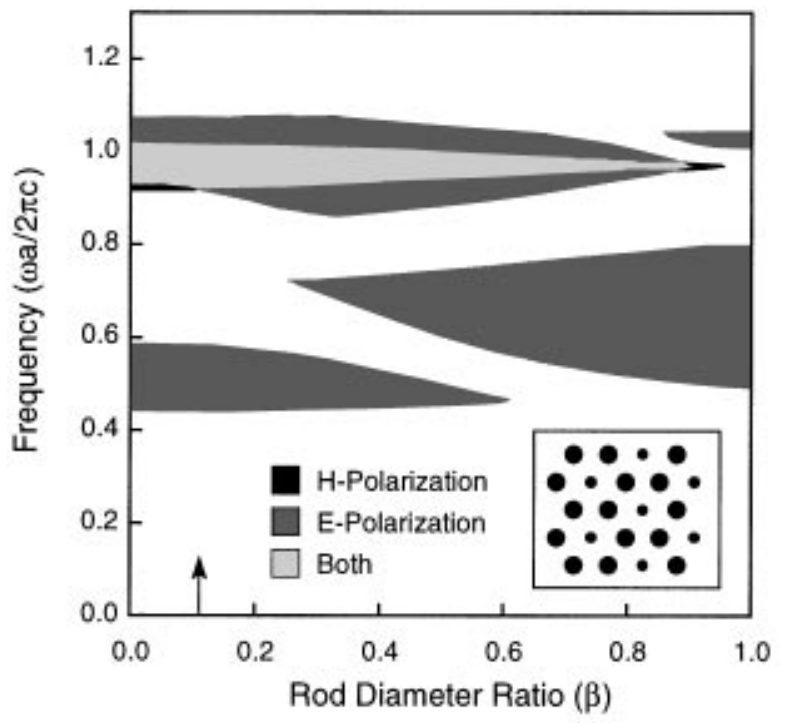

FIG. 5. Gap map for the reduced-symmetry triangular lattice $(f=0.14)$ of dielectric rods $\left(\epsilon_{a}=11.4\right)$ in air, as a function of $\beta$. The large honeycomb lattice gap (shown at $\beta=0$ ) can be increased further through symmetry reduction. The maximum absolute gap occurs at $\beta=0.11$ (arrow). 
without a significant reduction in the $H$-polarization gap. The largest absolute gap for the honeycomb lattice occurs at $f=0.14$, and has a magnitude $\Delta \omega=0.0880(2 \pi c / a)$. Increasing $\beta$ causes the gaps to shift as illustrated in the gap map of Fig. 5. The absolute band gap is always bounded at the top by the upper boundary of the $\mathrm{H}$ polarization gap. The lower boundary switches from $E$ to $H$ polarization. The maximum value of the absolute gap occurs at this crossover point, which for $f=0.14$ occurs at $\beta=0.11$. Here, the size of the absolute gap becomes $0.0967(2 \pi c / a)$, an increase of $9.9 \%$ over the largest gap for the honeycomb lattice.

Symmetry reduction also helps increase the size of the PBG at filling fractions different from that of the maximum for the honeycomb lattice. For example, Fig. 6 shows the gap map for the reduced-symmetry triangular lattice when $f=0.22$. For the honeycomb lattice $(\beta=$ 0 ) the absolute band gap at this filling fraction is much smaller than the maximum at $f=0.14$. However, the addition of a small $\operatorname{rod}(\beta=0.17)$ into the honeycomb cell center increases the absolute gap to $0.0849(2 \pi c / a)$, nearly double the size of that for the honeycomb lattice at the same filling fraction, albeit $12 \%$ smaller than the maximum gap observed in Fig. 5. This trend could be important when fabrication issues are considered. It may be beneficial to sacrifice a small amount of the band gap width in order to gain the ability to make more robust photonic crystals with larger filling fractions.

There is a remarkable similarity between the optimal rod diameter ratios for both the reduced-symmetry square and triangular lattices, emerging from a comparison of Figs. 4, 5 , and 6 . This similarity suggests that the lattice type may not be the critical factor in the creation of an absolute gap in

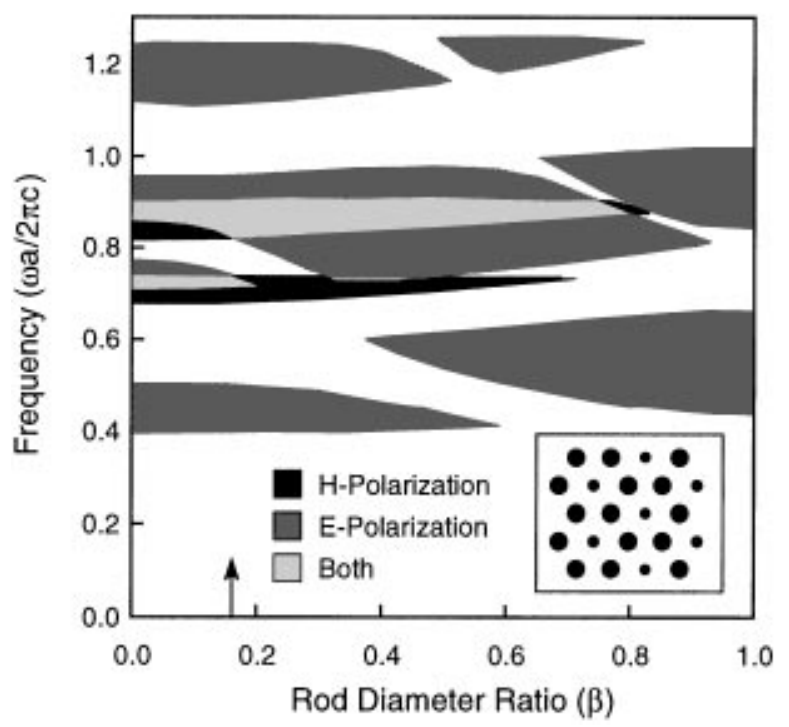

FIG. 6. Gap map for the reduced-symmetry triangular lattice $(f=0.22)$ of dielectric rods $\left(\epsilon_{a}=11.4\right)$ in air, showing a two-fold increase in the absolute gap size over the honeycomb lattice gap at the same filling fraction. The maximum absolute gap occurs at $\beta=0.17$ (arrow).
2D. Instead, the elements comprising the lattice may play a critical role in determining the maximum absolute gap that can be achieved for a given dielectric contrast. This observation is also supported by the work of Villeneuve and Piché in their study of the shape of the rod cross section [12]. Extending the concept of symmetry reduction to 3D crystals by changing the relative size of the elements comprising the lattice may also lead to increased 3D PBGs.

In conclusion, we have shown that symmetry reduction in both the 2D square and honeycomb lattices can increase the size of absolute photonic band gaps. Specifically, addition of a smaller rod in the center of a square lattice can yield photonic crystals with gaps significantly larger than the single-rod lattice with the same dielectric contrast. For air holes in a background medium of GaAs $(\epsilon=$ 11.4), the maximum gap of the reduced-symmetry square lattice was nearly 3 times the size of the best single-rod lattice band gap. The largest absolute band gap of the honeycomb lattice of dielectric rods in air can be increased by $\sim 10 \%$ also by inserting a small rod in the center of each unit cell. Crystal symmetry reduction opens up new vistas for engineering the band gap of $2 \mathrm{D}$ and possibly $3 \mathrm{D}$ crystals.

This work was partially supported by Caltech start-up funds and by a Camille and Henry Dreyfus Foundation New Faculty Award to K.P. G.

*To whom correspondence should be addressed.

[1] E. Yablonovitch, Phys. Rev. Lett. 58, 2059 (1987).

[2] R. D. Meade et al., Phys. Rev. B 44, 13772 (1991).

[3] D. L. Bullock, C. Shih, and R. S. Margulies, J. Opt. Soc. Am. B 10, 399 (1993).

[4] K. Inoueshi et al., Jpn. J. Appl. Phys. 33, L1463 (1994).

[5] U. Grüning et al., Appl. Phys. Lett. 68, 747 (1996).

[6] K. M. Ho, C. T. Chan, and C. M. Soukoulis, Phys. Rev. Lett. 65, 3152 (1990).

[7] P. R. Villeneuve and M. Piché, Phys. Rev. B 46, 4969 (1992).

[8] D. Cassagne, C. Jouanin, and D. Bertho, Nuovo Cimento D 17, 1401 (1995).

[9] International Tables for Crystallography, edited by T. Hahn (D. Reidel Publishing Company, Boston, 1987).

[10] Z. Zhang and S. Satpathy, Phys. Rev. Lett. 65, 2650 (1990).

[11] K. M. Leung and Y.F. Liu, Phys. Rev. Lett. 65, 2646 (1990).

[12] P. R. Villeneuve and M. Piché, Phys. Rev. B 46, 4973 (1992).

[13] R. D. Meade et al., Appl. Phys. Lett. 61, 495 (1992).

[14] M. Plihal et al., Opt. Commun. 80, 199 (1991).

[15] When $\beta=1$, the gap positions are shifted by a factor of $\sqrt{2}$ as a result of the change in the unit cell size.

[16] J. N. Winn, R. D. Meade, and J. D. Joannopoulos, J. Mod. Opt. 41, 257 (1994).

[17] J.D. Joannopoulos, R. D. Meade, and J. N. Winn, in Photonic Crystals (Princeton University Press, Princeton, 1995), p. 125. 